\title{
Rare Pulmonary Tumours: A Report of Two Cases with Diagnostic Challenge
}

\author{
Anita Harsh ${ }^{1 *}$, Deepika Hemrajani² \\ ${ }^{1 *}$ Associate professor, ${ }^{2}$ Assistant professor, \\ Department of Pathology, SMS Medical College, Jaipur, Rajasthan, India.
}

\begin{abstract}
Pulmonary blastoma and Pleuropulmonary blastoma are rare lung tumours with variable prognosis. These are now segregated in two different categories in the recent WHO 2015 classification of lung tumours. They required clinical, radiological, histopathological and immunohistochemical assistance for the diagnosis. The genetic studies are emerging as an important tool for diagnosis and as therapeutic target in future.
\end{abstract}

Key words: Pulmonary blastoma, Pleuropulmonary blastoma, Immunohistochemistry.

\section{Article History:}

Received: 19-09-2016, Revised: 04-10-2016, Accepted: 29-11-2016

\section{INTRODUCTION}

Pulmonary blastoma is a sarcomatoid neoplasm comprising 0.25 $-0.5 \%$ of all primary lung tumours. ${ }^{1}$ Over 200 cases have been reported till date worldwide since the first description of Barrett and Barnard in $1945 . .^{2}$ The usual age of presentation is in fourth decade of life. This is a biphasic tumour comprising of malignant primitive epithelial and mesenchymal components and the tumour resembles fetal lung between 10-16 weeks of gestation.

Pleuropulmonary blastoma is a rare mesenchymal neoplasm. Over 350 cases have been registered till now. ${ }^{3}$ Median age of presentation is 2 years and the tumour comprises of malignant embryonic appearing mesenchyme with no epithelial or entrapped benign epithelial component.

We here report 2 cases of the above mentioned rare tumours presenting at an unusual age.

\section{CASE PRESENTATION \\ CASE NO 1}

A 23 year old male, non-smoker presented with dyspnea on exertion for last 2 months. CECT revealed hypodense area in anterior mediastinum extending till right hilum. The mass was abutting brachiocephalic vein, aorta, superior vena cava, right branch of pulmonary artery and cardiac chambers with cardiac displacement and compression.

The therapeutic thoracotomy was done. The right pneumonectomy specimen with tumour mass was received. Right

\section{${ }^{*}$ Correspondence to:}

\section{Dr. Anita Harsh,}

Associate professor

Department of Pathology,

S. M. S. Medical College,

Jaipur, Rajasthan, India.

\begin{tabular}{|l|c|}
\hline \multicolumn{2}{|c|}{ Access this article online } \\
\hline $\begin{array}{l}\text { Website: } \\
\text { www.ijmrp.com }\end{array}$ & Quick Response code \\
\hline DOl: & \\
10.21276/ijmrp.2016.2.6.045 & \\
\hline
\end{tabular}

lung measured $18 \times 18 \times 4 \mathrm{~cm}$ and the tumour mass measured $34 \times 21 \times 13 \mathrm{~cm}$. The tumour was covered by pleura, with breech of pleura at places. Tumour was originating from the middle lobe of right lung with variegated gray white, congested, fleshy areas and extensive necrosis on cut section.

Microscopically, tumour revealed tubular structures lined by pseudostratified columnar epithelium. Cells having eosinophilic cytoplasm, subnuclear and supranuclear vacuolation giving the glands an endometrioid appearance resembling fetal lung tissue in pseudoglandular stage. In addition, cords, solid nests with basal palisading, small rosette like glands and occasional morules were seen.

Blastemal stroma comprised of small oval and spindle shaped cells in a myxoid background. At places the cells were condensed around neoplastic glands. Occasional giant cells, mitoses, pleomorphism in spindle cells and mixed inflammatory infiltrate in focal areas noted. Extensive areas of haemorrhage, necrosis and areas of cartilage differentiation were seen. The attached lymph nodes were free from tumour. No lymphovascular invasion seen. Immunohistochemical studies showed positivity for cytokeratin, EMA and CEA in neoplastic glands. Focal chromogranin positivity was observed in morules and glands.

Malignant mesenchymal component showed vimentin and SMA positivity. A diagnosis of biphasic pulmonary blastoma was made on histopathology and immunohistochemistry. 


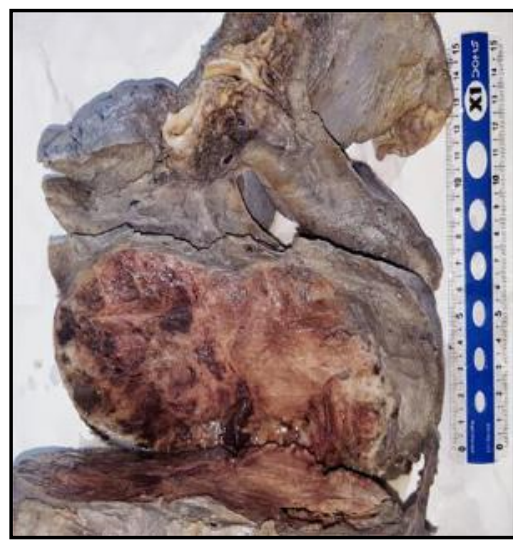

Fig. 1A- Tumour arising from lung

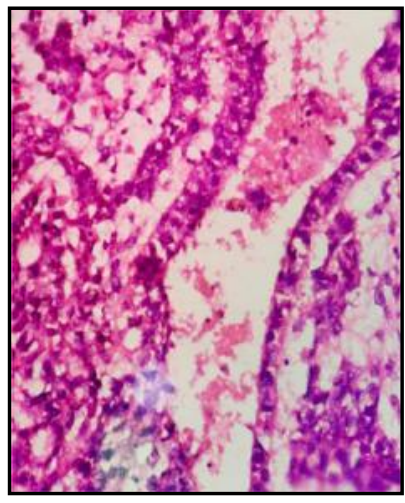

1C - Tubule lined by pseudostratified columnar epithelium (H\&E, 100X)
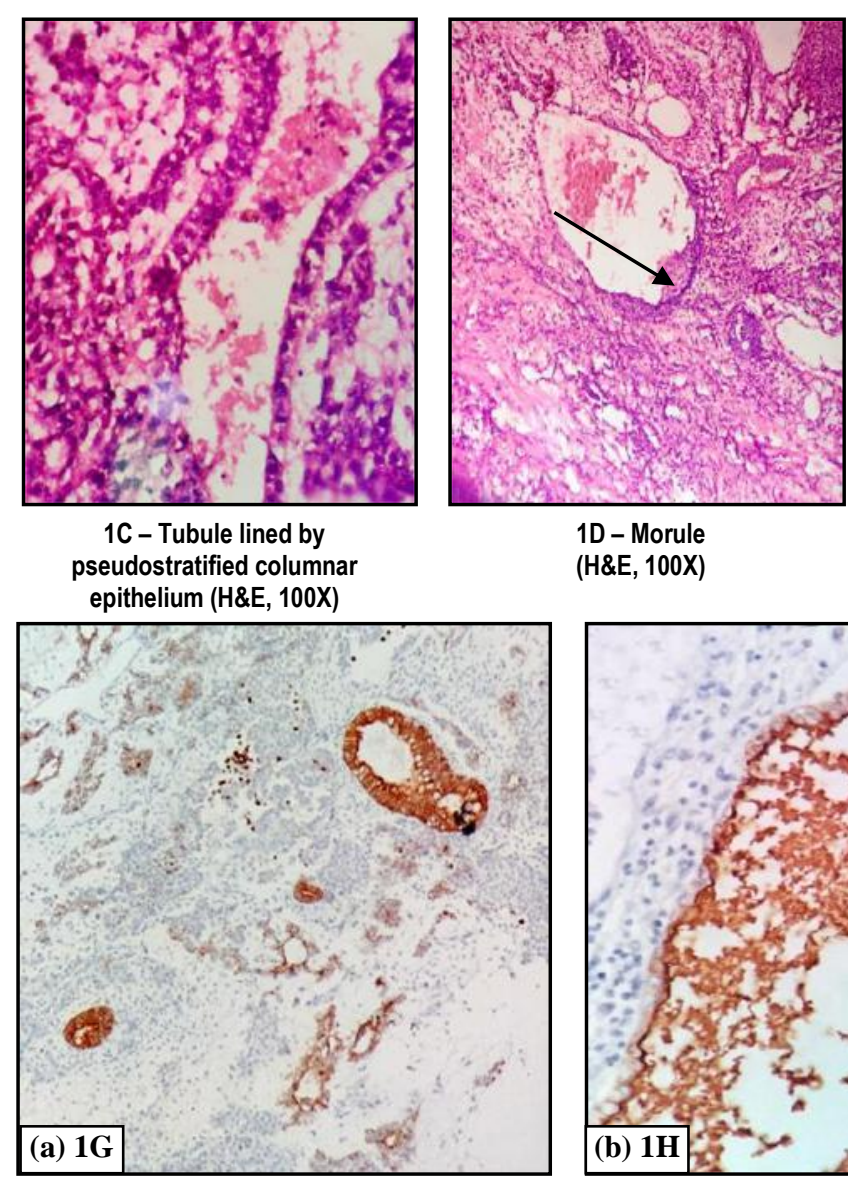

1D - Morule (H\&E, 100X)

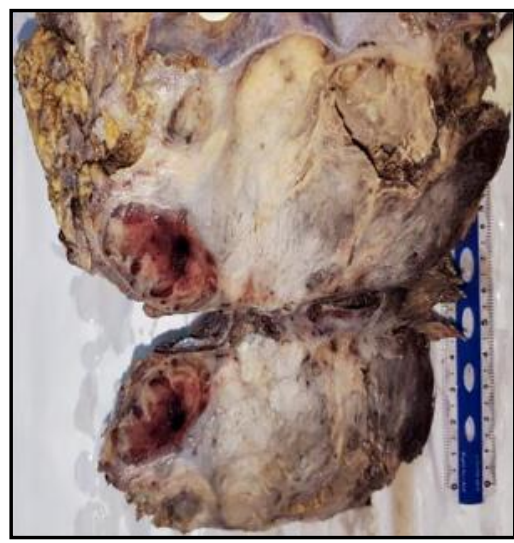

Fig. 1B- Variegated cut surface

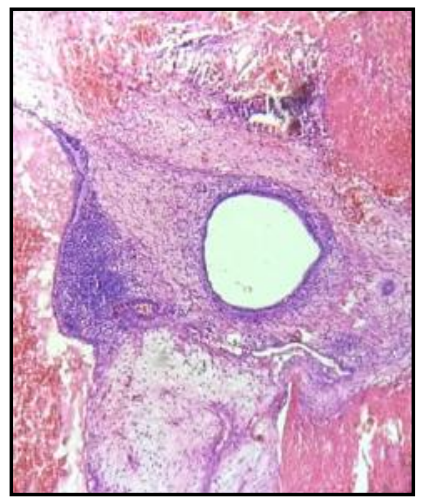

$1 \mathrm{E}$ - Blastemal stroma in a myxoid background, haemorrhage and necrosis (H\&E, 100X)
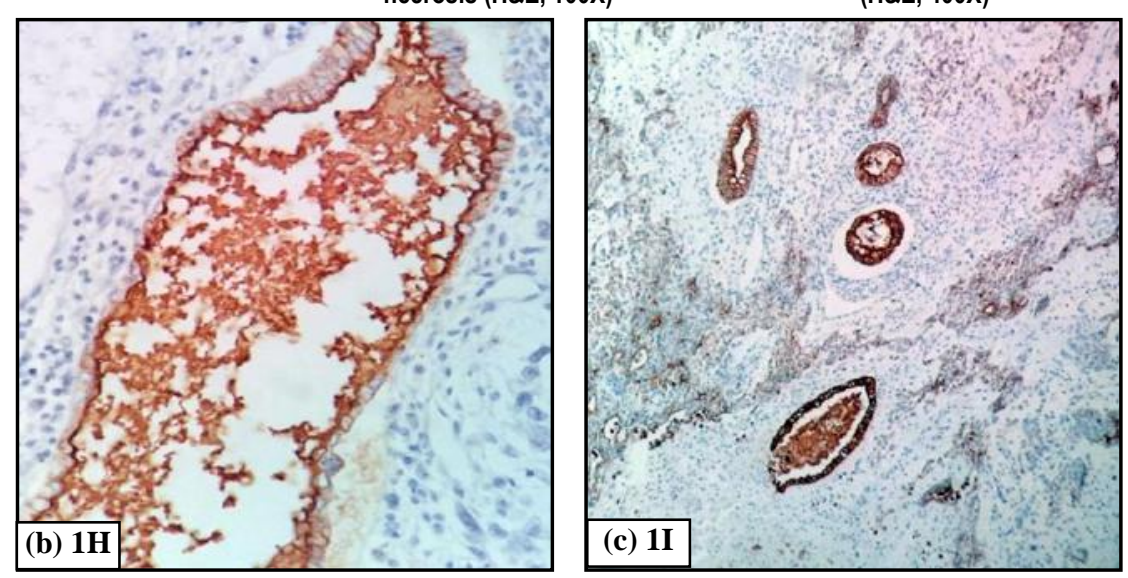

Immunostain Positivity in Neoplastic Glands (100X)

(a) 1G-Cytokeratin; (b) $1 \mathrm{H}$ - Carcino-Embryonic Antigen; (c) $1 \mathrm{I}$ - Epithelial Membrane Antigen
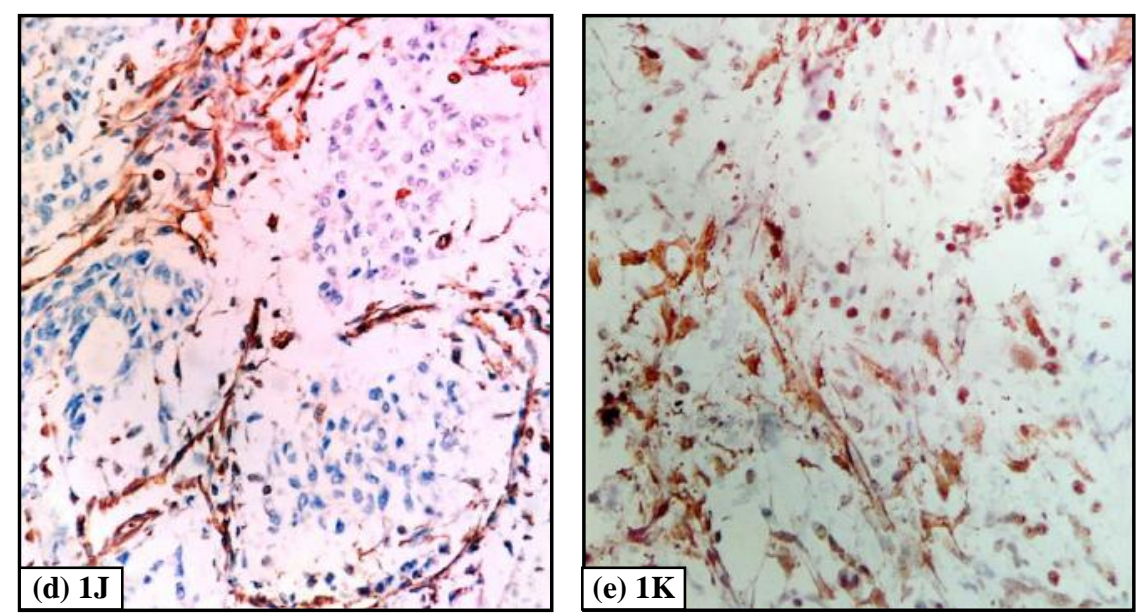

Immunostain Positivity in Mesenchymal Components (100X)

(d) 1J-Vimentin; (e) 1K- SMA 


\section{CASE NO 2}

A 15 year old male presented with progressive dyspnea. There was no other associated symptom. CECT revealed a large well defined predominantly cystic mass lesion with patchy small solid component inferiorly in right hemithorax. The mass measured $13.2 \times 12.4 \times 10.5 \mathrm{~cm}$. Moderate amount of right sided pleural effusion was seen.

Excised tumour was received in several pieces consisting of grey brown to grey black soft tissue pieces measured collectively $15 \times 10 \mathrm{~cm}$. Some of the pieces appeared flattened and others congested. Congested areas of lung parenchyma and blood clots were seen.
On microscopic examination, several solid and cystic components were seen. The cystic component showed low cuboidal epithelium. Both solid and cystic components showed small round cell with moderate amount of eosinophilic cytoplasm, hyperchromatic nuclei and frequent mitoses. The primitive mesenchymal tissue along with extensive areas of hemorrhage, necrosis and acute inflammatory cells infiltrate was seen. At places perivascular rosettes also noted. IHC studies showed strong positivity for vimentin and SMA. However entrapped benign epithelium showed diffuse cytoplasmic positivity for cytokeratin. A diagnosis of Pleuropulmonary blastoma was given.

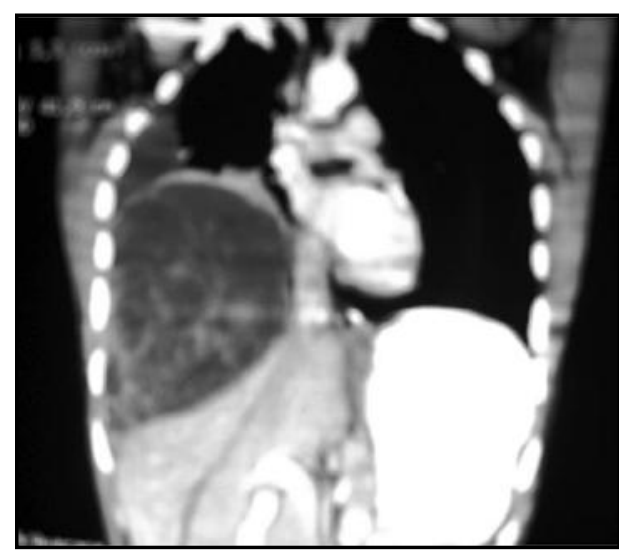

2A - CECT chest - A large well defined predominantly cystic mass with patchy small solid components in right hemithorax

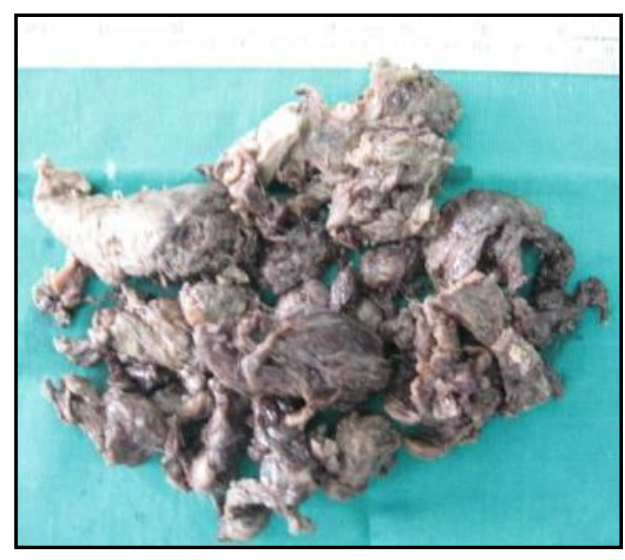

2B- Gross appearance- Several irregular, flattened and congested tissue pieces

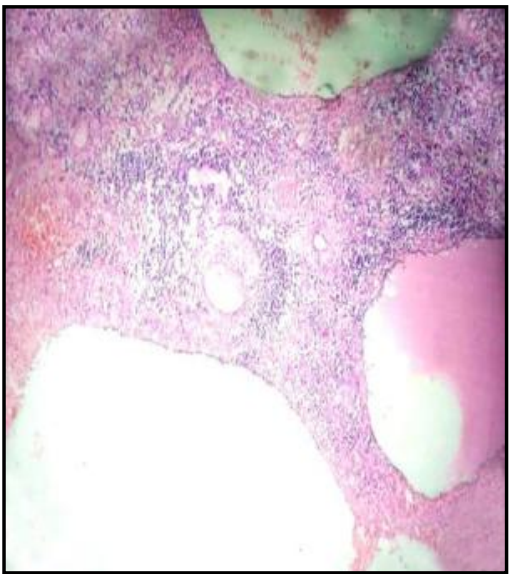

2C - Cystic and solid components (H\&E, 100X)

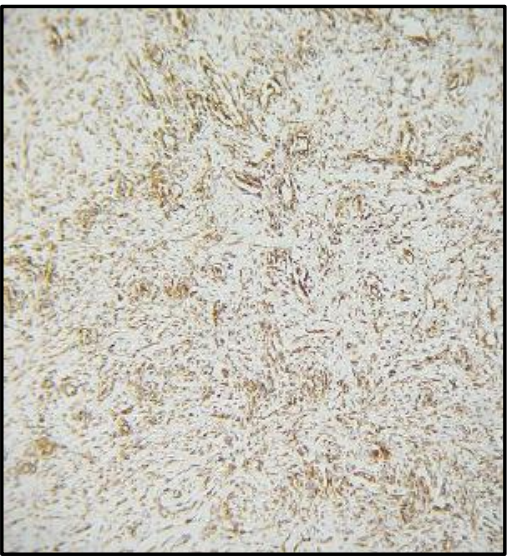

2G- Mesenchymal component showing positivity for vimentin (100X)

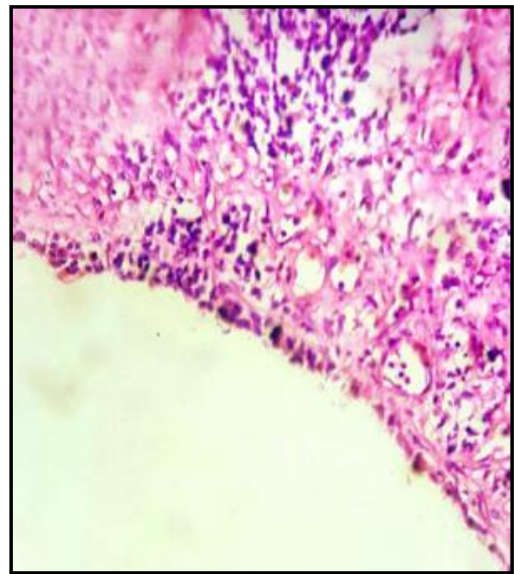

2D- Cystic component lined by low cuboidal epithelium (H\&E, 100X)

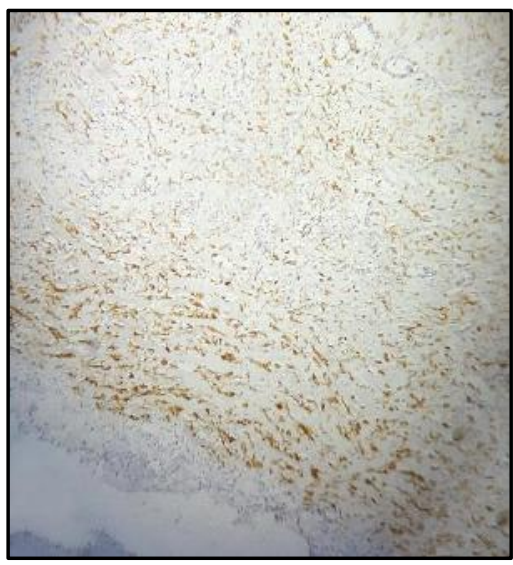

2F- Mesenchymal component showing positivity for SMA (100X)

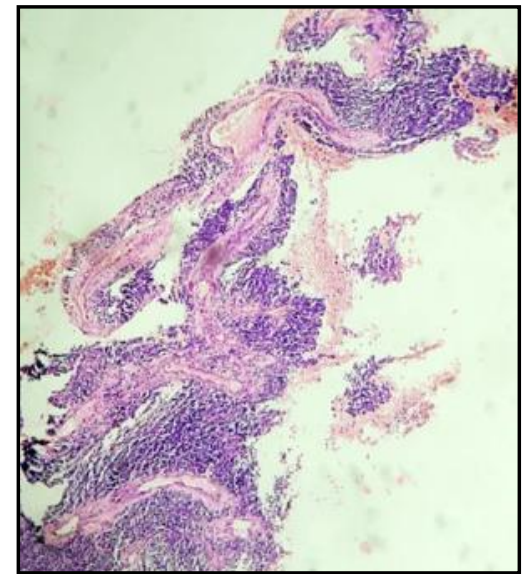

2E- Blastemal component (H\&E, 100X)

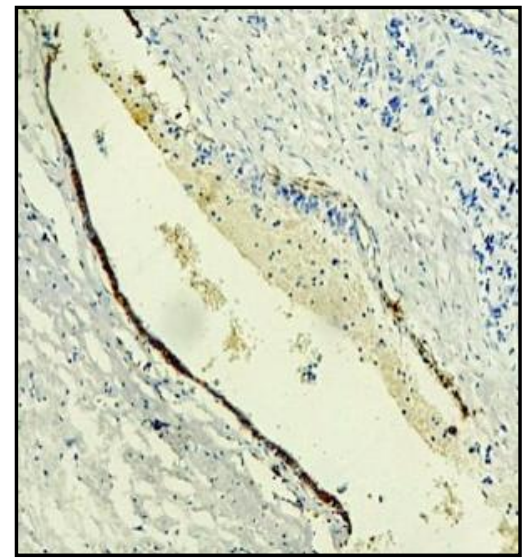

$2 \mathrm{H}$ - Entrapped benign epithelium showing cytokeratin positivity (100X) 


\section{DISCUSSION}

Pulmonary blastoma is a rare, aggressive neoplasm of lung that has been classified as sarcomatoid type of epithelial neoplasm according to WHO 2015 classification of lung tumours. ${ }^{4}$

PB typically presents with cough, dyspnea, hemoptysis and chest pain in fourth decade of life. Smoking is an associated risk factor. This rare tumour has got poor prognosis. It almost always presents as solitary, well demarcated, unencapsulated mass on chest radiograph.

Mutation in CTNNB1 gene leads to constitutive activation of WNT signaling pathway through aberrant nuclear / cytoplasmic localization of $\beta$ catenin. Immunostaining for $\beta$ catenin, hence is a useful marker to distinguish pulmonary blastoma from high grade fetal adenocarcinoma and sarcomatoid variant of carcinosarcoma. ${ }^{5}$

Maeccher Goeppinger S. et al observed CTNNB1 gene sequencing is useful in distinguish PB from other lung cancers and also observed that EGFR expression is present in all pulmonary blastomas. There was co-existence of EGFR and $\beta$ catenin mutation. 6

Our case no.1 was a young non-smoker male presented with dyspnea on exertion for two months. Chest radiography is usually helpful. ${ }^{4}$ In our patient CECT chest also showed hypodense area in anterior mediastinum extending till right hilum with encasement of all major vessels, cardiac displacement and compression.

Immunohistochemistry is essential to confirm the biphasic components by using combination of epithelial and mesenchymal markers. ${ }^{4}$ In our case, IHC panel of CK, EMA, CEA, vimentin, desmin, S-100, SMA was applied that confirmed malignant epithelial and mesenchymal components consistent with biphasic pulmonary blastoma.

Currently according to WHO 2015 classification of lung tumours, Pleuropulmonary blastomas are classified as mesenchymal neoplasm. PPB usually present as respiratory distress with or without pneumothroax. 3 basic pathologic types are recognized. Type-I is being predominantly cystic. Type-II PPB is partly solid and partly cystic. Type-III is almost entirely solid, firm to gelatinous creamy white tumours.

Type-I has more favourable prognosis than Type-II and III. Local recurrence and predilection for metastasis to brain and skeletal system is seen.

Type-I PPB are characterized by cystic structure, lined by respiratory type of epithelium and a cambium layer like zone of small primitive malignant cells. These cells may show rhabdomyoblastic differentiation. Solid areas of Type-II and TypeIII PPB have mixed sarcomatous and blastomatous features with aggregates of anaplastic stromal cells, fibrosarcoma like areas or rhabdomyoblasts.

Ronald $\mathrm{R}$ de Krizer et al demonstrated frequent gain of chromosome 8q in PPB. p53 mutations, unbalanced translocation between chromosome 1 and $X$, loss of chromosomal 9p21-24 and del 11 p14 are other genetic alterations. ${ }^{7}$

Our case no. 2 was a 15 years old male patient. CECT revealed a large well defined predominantly cystic mass lesion with patchy small solid components.

IHC \& genetic studies are helpful in diagnosis. EMA, CK, CD99 were negative unlike synovial sarcomas and PPB showed vimentin, SMA, desmin and S-100 positivity. Histopathology and
IHC findings were consistent with diagnosis of pleuropulmonary blastoma.

Both tumours pose diagnostic problem as they cannot be diagnosed only on histopathology.

It requires correlation of clinical, radiological, histopathology and genetics for diagnosis and differentiation from other lesions, however the genetic analysis is limited to the research laboratories.

Surgery is an optimal treatment for localized disease. Most cases do not show response to radiotherapy. ${ }^{8}$ Prognosis is poor with 5 year survival rate of $16 \%$ only in cases of pulmonary blastoma. Overall survival for Type -I pleuropulmonary blastoma is $80-85 \%$ and for type II and type III is $45-50 \%$. Large size of tumour, tumour metastasis and tumour recurrence predict poor prognosis. $43 \%$ of tumour recurs within a year with metastasis to brain and mediastinum. $^{9}$

\section{CONCLUSION}

Pulmonary blastoma is a rare aggressive sarcomatoid type of epithelial neoplasm of lung, usually occurs in about 40 years of age and carries poor prognosis with 5 years survival rate of $16 \%$.

Pleuropulmonary blastoma is also a rare mesenchymal neoplasm of lung occurring in children less than 10 years of age. The three PPB types carry variable prognosis.

Both tumours are rare to present at young age and must always be kept as differential in mind. Till date surgery is the mainstay of treatment, but with the emergence of genetics the selection of therapy in an individual case may be based on the histopathology, genetic analysis and stage of the tumour. The targeted therapy may hold future promise.

\section{SOURCE OF SUPPORT: Nil.}

\section{CONFLICT OF INTEREST: None Declared.}

\section{REFERENCES}

1. Cutler C.S., Michel R.P., Yassa M. and Langleben A. Pulmonary blastoma: case report of a patient with a 7 year remission and review of chemotherapy experience in the world literature. Cancer, 1998; 82(3); 462-467.

2. Barrett N.R. and Barnard W.G. Some unusual thoracic tumours. British Journal Surgery, 1945; 32(128):447-457.

3. Messinger Y.H., Steward D.R., Priest J.R. et al. Pleuropulmonary blastoma : A report on 350 central pathology confirmed pleuropulmonary blastoma cases by the international pleuropulmonary blastoma registry. Cancer, 2015; Jan15;121(2); 276-285.

4. Travis W. D., Brambilla E., Nicholson A. G. et al. The 2015 WHO classification of lung tumors. Impact of genetic, clinical and radiologic advances since the 2004 classification. Journal of Thoracic Oncology, 2015; vol 10, no. 9; sept 2015; 1243 1260.

5. Nakatani $Y$, Miiyagi $Y$, Takemura $T$ et al. Aberrant nuclear/cytoplasmic localization of gene mutation of $\beta$ catenin in classic pulmonary blastoma. $\beta$ catenin immunostaining is useful for distinguishing between classic pulmonary blastoma and 
blastomatoid variant of carcinosarcoma. Am. J. Surg. Pathol., 2004; 28(7):921-927.

6. Macher Goeppinger S, Penzel R, Roth W et al. Expression and mutation analysis of EGFR, C-KIT and $\beta$ catenin in pulmonary blastoma. J. Clin. Pathol, 2011;64(4):349-353.

7. Ronald R. de Krijger, Sandra M. H., Claesson et al. Gain of chromosome $8 \mathrm{q}$ is a frequent finding in pleuropulmonary blastoma. Modern Pathology, 2007; 20: 1191-1999.

8. Larsen H, Sorensen J.B. Pulmonary blastoma: A review with special emphasis on prognosis and treatment. Cancer Treat Rev., 1996; 22:145-160.

9. Koss M.N., Hochholzer L., O' Leary T. Pulmonary blastoma. Cancer, 1991; 67: 2368-2381.

10. Indolfi $P$, Casale F, Carli M.et al. Pleuropulmonary blastoma: Management and prognosis of 11 cases. Cancer, 2000; 89(6):1396-1401.
Copyright: (c) the author(s) and publisher. IJMRP is an official publication of Ibn Sina Academy of Medieval Medicine \& Sciences, registered in 2001 under Indian Trusts Act, 1882.

This is an open access article distributed under the terms of the Creative Commons Attribution Non-commercial License, which permits unrestricted non-commercial use, distribution, and reproduction in any medium, provided the original work is properly cited.

Cite this article as: Anita Harsh, Deepika Hemrajani. Rare Pulmonary Tumours: A Report of Two Cases with Diagnostic Challenge. Int J Med Res Prof. 2016; 2(6):222-26.

DOI:10.21276/ijmrp.2016.2.6.045 